\title{
Abdominal Ectopic Pregnancy and Impaired Postnatal Mammary Gland Development, Consistent With Physiologic Agalactia, in a Wild European Rabbit, Oryctolagus cuniculus
}

\author{
Katherine Hughes* \\ Department of Veterinary Medicine, University of Cambridge, Cambridge, United Kingdom
}

OPEN ACCESS

Edited by:

Robert James Ossiboff, University of Florida, United States

Reviewed by:

Martha Ann Delaney,

University of Illinois at

Urbana-Champaign, United States

Steven H. Weisbroth,

Consultant, McLean, VA,

United States

John Ford Roberts,

University of Florida, United States

*Correspondence: Katherine Hughes

kh387@cam.ac.uk orcid.org/0000-0002-3331-1249

Specialty section: This article was submitted to Veterinary Experimental and

Diagnostic Pathology,

a section of the journal

Frontiers in Veterinary Science

Received: 22 May 2019

Accepted: 17 July 2019 Published: 07 August 2019

Citation:

Hughes K (2019) Abdominal Ectopic

Pregnancy and Impaired Postnatal

Mammary Gland Development, Consistent With Physiologic Agalactia, in a Wild European Rabbit,

Oryctolagus cuniculus.

Front. Vet. Sci. 6:254. doi: 10.3389/fvets.2019.00254
A wild European rabbit, Oryctolagus cuniculus, was diagnosed with an abdominal pregnancy due to the presence of a single abdominal lithopedion attached by a thin fibrovascular stalk to the left uterine horn, which was distorted by the tension of the adhesion. Evidence of mineralized remnants, chronic inflammation, and fibrosis in the left uterine endometrium and myometrium suggested that the lithopedion had arisen as a secondary abdominal pregnancy. The right uterine horn contained two macroscopically normal fetuses. The mammary gland exhibited notably retarded development in relation to the size of the fetuses. Histologically, mammary alveoli lacked evidence of intraluminal secretory product, and ducts lacked prominence and contained clusters of small numbers of macrophages. The doe also exhibited mild granulomatous and heterophilic pneumonia with rare intralesional adiaspores, suggesting infection with Emmonsia spp. as an incidental finding. This case documents secondary abdominal pregnancy in a wild lagomorph not subjected to artificial insemination procedures suggested to increase the occurrence of this condition in farmed rabbits. An abdominal pregnancy is one of a number of factors that should be considered as a potential factor in the etiology of impaired postnatal mammary development or reduced milk yield in a breeding doe, although no causative association is demonstrated in this case. Abdominal ectopic pregnancy is one possible differential diagnosis in the investigation of the presence of a palpable abdominal mass or masses in O. cuniculus.

Keywords: abdominal pregnancy, ectopic pregnancy, extrauterine pregnancy, lithopedion, mammary gland, Oryctolagus cuniculus, pregnancy, rabbit

\section{BACKGROUND}

Extrauterine pregnancy, often used synonymously with the term "ectopic pregnancy," describes a pregnancy occurring outwith the uterus (1). Four subtypes are recognized (Table 1) but the most common subtype arising in animals, particularly in species other than non-human primates, is abdominal pregnancy. Primary abdominal pregnancy arises when the conceptus implants within the abdomen due to escape from the fallopian tube, for example following retrograde movement 
TABLE 1 | Tabular representation of subtypes of extrauterine pregnancy, as recognized in human medicine, with selected case examples from humans and animals.

Extrauterine pregnancy

Often used synonymously with the term "ectopic pregnancy" to describe a pregnancy occurring outwith the uterus (1).

\section{Tubal}

The fertilized ovum implants in the fallopian tube, most frequently in the ampulla in humans (2, 3).

\section{Ovarian}

The ovary is the site of implantation, growth, and development of the gestational sac (4).

\section{Cervical}

The site of implantation is the cervical canal-a rare form of ectopic pregnancy in humans (5).

\author{
Abdominal [peritoneal] \\ The fertilized ovum implants in the abdomen. The \\ most common subtype of ectopic pregnancy \\ recognized in animals other than non-human \\ primates (1).

Primary
$\begin{aligned} & \text { Abdominal implantation is } \\ & \text { due to the fertilized ovum orine or tubal trauma } \\ & \text { entering the peritoneal }\end{aligned}$
leavity, such as through the oocyte release into
fimbria. Lack of lesions in
the reproductive tract is
suggestive of primary
extrauterine pregnancy, but
the possibility of repair
cannot be discounted (6).

through the fimbria. By contrast, secondary abdominal pregnancy occurs following uterine or tubal trauma that results in abdominal implantation.

Abdominal pregnancies have been previously described in laboratory (8), pet (9-11), and farmed Oryctolagus cuniculus (1, $12,13)$. Indeed, in farmed rabbits, use of artificial insemination has been postulated to be a factor in the pathogenesis of some cases (12). Abdominal pregnancy in wild lagomorphs is likely under-reported, although two cottontail rabbits (Sylvilagus floridanus) (14), two European hares (Lepus europaeus) (15), and a mountain hare (Lepus timidus scoticus) (16) are amongst the reports of abdominal pregnancies in this group of animals. The present report describes a case of secondary abdominal pregnancy in a wild European rabbit, O. cuniculus, with concurrent impaired mammary gland development.

\section{CASE PRESENTATION}

The cadaver of a wild female European rabbit, O. cuniculus, was one of a group shot for population management and donated for research and teaching to the veterinary anatomic pathology service of the Department of Veterinary Medicine, University of Cambridge (UK). The rabbit weighed $1.78 \mathrm{~kg}$. Post mortem examination revealed a very firm, irregular mass with a smooth, gray/pink surface in the left abdomen. The mass had average dimensions $40 \times 35 \times 30 \mathrm{~mm}$ and had thin, tough, translucent adhesions to the left abdominal wall and the left uterine horn, the latter adhesion exhibiting evidence of vascularity, and the tension of the adhesion causing distortion of the left uterine horn (Figures 1A,B). On cut section, the mass was dry and multifocally gritty, but contained clearly discernable fetal remnants, including skin, hair, hard palate, and bone surrounded by lamellated fibrous tissue and fetal membranes (Figure 1C). The mass was interpreted to be a lithopedion, or mineralized abdominal ectopic pregnancy (17).

At the point of attachment to the left uterine horn, the uterine lumen contained an irregular, cream, $12 \times 8 \times 5 \mathrm{~mm}$ concretion and a circumferential $10 \mathrm{~mm}$ band of the uterine endometrium was dark red at this focus. The right uterine horn contained two fetuses, with crown-rump lengths 55 and $53 \mathrm{~mm}$. Macroscopically, the mammary gland exhibited some evidence of development consistent with early pregnancy, such as prominent vasculature and mild parenchymal development, but this was considered markedly less than expected for this stage of pregnancy based on previous analyses (18). The gastrointestinal tract, in particular the stomach, was subjectively less full than the majority of wild rabbits from the same region examined as part of ongoing studies $(18,19)$ and scarce fecal pellets were present in the rectum. No other abnormalities were detected macroscopically.

Tissues were fixed in $10 \%$ neutral buffered formalin, and histological sections were prepared following standard procedures. The lithopedion was composed of amorphous eosinophilic material, indicative of degeneration and autolysis, which was multifocally heavily mineralized. Although nuclei were multifocally apparent, the degree of degeneration was such that cell populations were not identifiable and the presence of an inflammatory infiltrate could not be assessed. The uterus at the point of attachment of the lithopedion exhibited a locally extensive endometrial infiltrate of moderate numbers of lymphocytes, plasma cells, heterophils, and fewer macrophages, with locally extensive ulceration. Underlying this focus, there was a loss of distinction of the myometrial layers and locally extensive, mild to moderate, fibrosis. Together with the presence of the small concretion of mineralized material within the uterine lumen, this was considered strong evidence of abdominal pregnancy and formation of a lithopedion secondary to uterine rupture.

The macroscopic impression that the mammary gland was markedly under-developed for the stage of pregnancy was confirmed histologically, with only a thin layer of mammary tissue present and mammary lobules interspersed by prominent, wide, bands of connective tissue. The mammary alveoli frequently lacked intraluminal proteinaceous material and ducts 


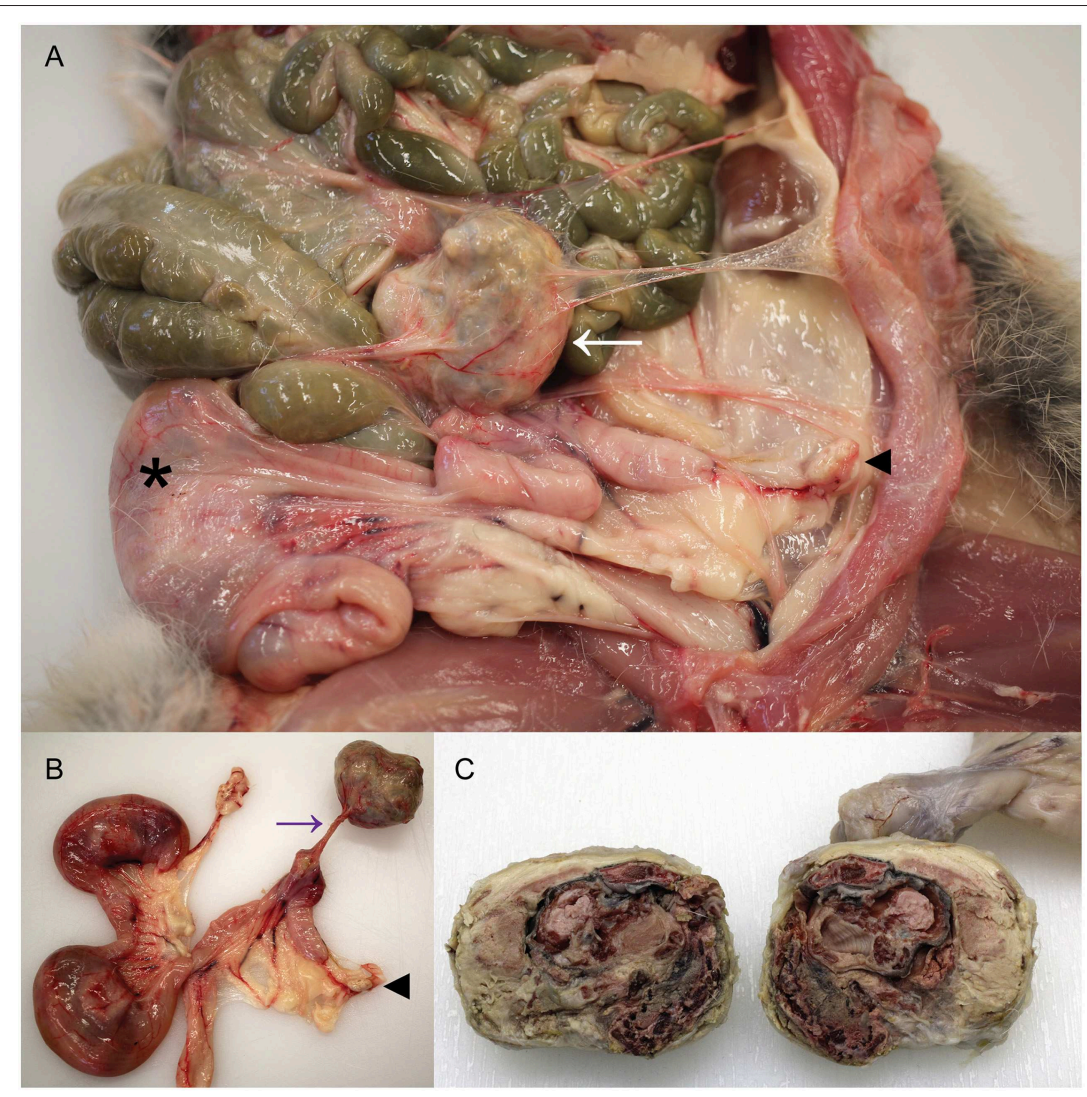

FIGURE 1 | Presence of a lithopedion in a wild European rabbit. (A) A firm mass with a smooth surface is present in the abdomen of a female rabbit (white arrow). Asterisk denotes fetus in right uterine horn; arrowhead indicates left ovary. (B) A thin fibrovascular band (purple arrow) connects the lithopedion to the left uterine horn, which is distorted as a result. Arrowhead indicates left ovary. (C) On cut surface the lithopedion contains fetal remnants including skin, hair, bone, and hard palate (partially fixed tissue).

were generally empty except for small numbers of macrophages and desquamated epithelial cells. The lack of mammary development was particularly pronounced when compared with mammary tissue from another female carrying a litter of similarly developed fetuses (crown-rump length median: $51 \mathrm{~cm}$; mean: $50 \mathrm{~cm} ; n=8$; Figure 2).

Histologically the lungs exhibited multifocal, mild, granulomatous and heterophilic pneumonia with multinucleated giant cells and very rare adiaspores, with morphology consistent with Emmonsia spp. There were no other significant findings on histological examination.

\section{DISCUSSION}

This report describes a case of secondary abdominal pregnancy in a wild rabbit. Secondary abdominal pregnancy is considered to arise as a consequence of traumatic injury to the reproductive tract, leading to a communication between the uterus or vagina and the peritoneal cavity, and the subsequent propulsion of a conceptus or fetus into the abdomen, potentially as a result of contractions associated with parturition in some cases $(7,12)$. In this case, the lithopedion was a similar size to the fetuses in the unaffected right uterine horn. It is therefore tempting to speculate that, given the degree of fetal maceration, the abdominal pregnancy originally arose during a previous pregnancy. This scenario is also consistent with the lack of detectable communication between the left uterine horn and the peritoneal cavity, and the presence of chronic inflammation and fibrosis in the myometrium suggestive of a chronic reparative process. The mineralized concretion within the uterus at this site likely represented residual material associated with the fetus expelled into the abdomen.

Studies in farmed or laboratory rabbits, for which dates of insemination are recorded, have facilitated accurate description of concurrent abdominal pregnancies and intrauterine fetuses of differing ages (13), suggesting that it is indeed feasible that the abdominal fetus described in this report originally arose during a previous pregnancy. This raises the question of whether the wild rabbit described here would have exhibited clinical signs associated with the presence of the lithopedion. Interestingly, during post mortem examination, it was felt that the stomach was less full than usual for rabbits examined as part of a cohort culled for population management, and the rectum was largely devoid of feces. It is therefore tentatively suggested that the lithopedion and associated adhesions were impacting the rabbit to a degree, supporting previous assertions that abdominal pregnancy may be associated with clinical signs in some cases: one pet rabbit diagnosed with an abdominal pregnancy was 


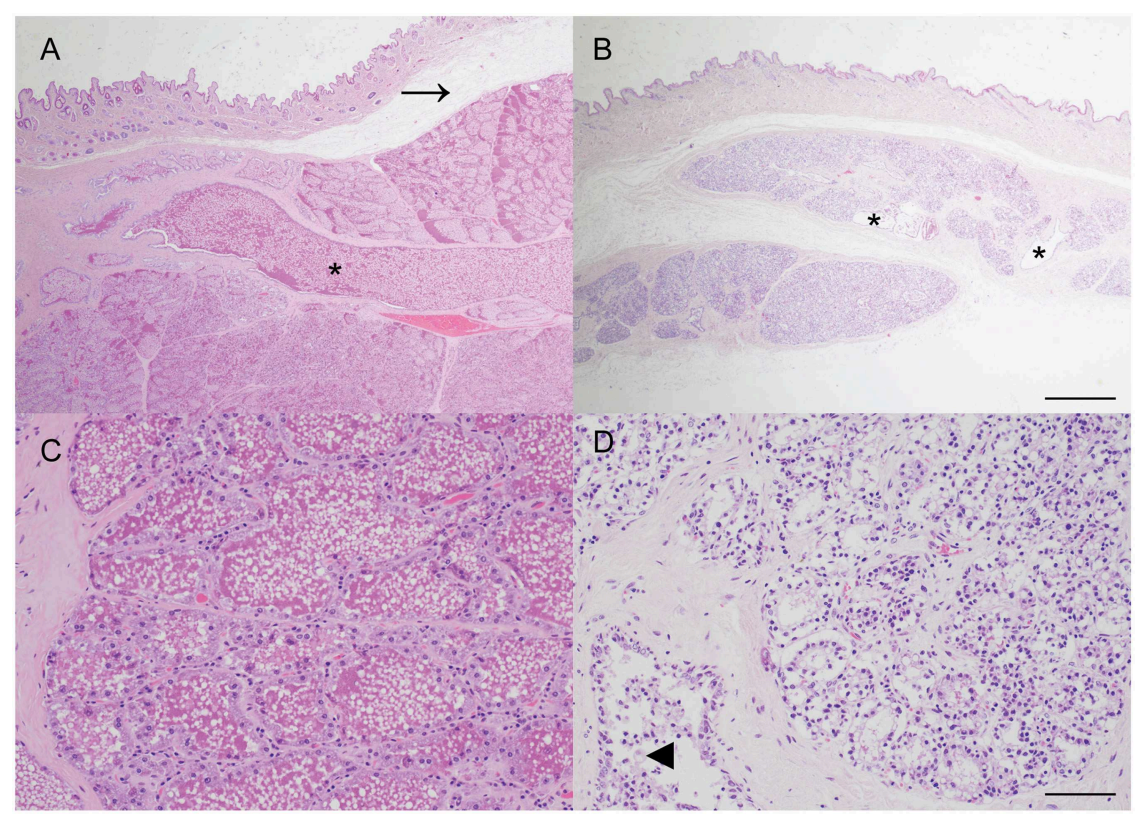

FIGURE 2 | Impaired postnatal mammary development, consistent with physiologic agalactia, in a wild European rabbit with an abdominal pregnancy. (A,C) Mammary tissue from a doe with fetuses of a similar gestational age to the affected doe exhibits extensive alveolar development, and large amounts of eosinophilic proteinaceous material within mammary alveoli and ducts (asterisk). Arrow indicates artifactual separation of skin from underlying mammary tissue. (B,D) Mammary tissue from a female rabbit with an abdominal pregnancy exhibits alveoli with reduced diameter and lacking secretion, and small, largely empty ducts (asterisks). Some ducts contain small clusters of macrophages (arrowhead). Haematoxylin and eosin stain. Scale bars indicate 800 microns (A,B) and 80 microns (C,D).

presented with inappetance and lethargy and subsequently died. Three mummified abdominal fetuses were detected during a post mortem examination, and although no definitive association with the death of the rabbit was described, no alternative cause of death was discovered (9). A further laboratory rabbit later discovered to have multiple abdominal lithopedions also exhibited anorexia (8). However, in another case in a pet rabbit, no clinical signs were noted (10).

Although no definitive relationship to the abdominal pregnancy can be inferred from this case, it is tempting to speculate on a potential relationship between the presence of the lithopedion and the impaired mammary development. Agalactia has been previously described in a laboratory rabbit with an extrauterine pregnancy although no description of the mammary gland was provided (8). In the present case, the relative lack of mammary development, when compared to an unaffected doe pregnant with similarly sized fetuses, is striking (Figure 2). One limitation of this case is that as the rabbit was wild, no reproductive history or details regarding concurrent lactation are available. The lack of postnatal mammary development, consistent with physiologic agalactia, may be attributable to a postulated lack of concurrent lactation due to loss of the previous litter, or the presence of just two viable fetuses, but it also seems possible that stress, resulting from the abdominal pregnancy and leading to elevated blood cortisol, may have played a more direct role. Secretion of prolactin, a key regulator of development of the milk secreting mammary epithelial cells via activation of Signal Transducer and Activator of Transcription 5 (STAT5) (20), is reduced in a rabbit model of critical illness using deep dermal burns (21). The impact of other stressors, such as heat stress, on postnatal mammary gland development are widely recognized in production ruminants (22). In this case there was no evidence of inflammation within the mammary gland and thus an infectious cause of agalactia was considered unlikely. This case highlights that further investigation of a potential link between abdominal pregnancy and impaired mammary gland development in rabbits may be merited.

The rabbit in this report was microscopically diagnosed with mild granulomatous and heterophilic pneumonia with multinucleated giant cells and very rare adiaspores, with morphology consistent with Emmonsia spp. We have recently described adiaspiromycosis in a wild European rabbit with a much higher burden of adiaspores and marked granulomatous pneumonia which was grossly apparent (19). By comparison, in this case the pathology was subtle, only appreciable histologically, and was considered to be most likely an incidental finding of no clinical consequence. Such incidental diagnosis of adiaspiromycosis has also been reported in other small mammal species such as moles (Talpa europaea) (23). DNA extraction and subsequent PCR amplification and sequencing would have been the optimal approach to attempt to definitively confirm the diagnosis of adiaspiromycosis in this rabbit, particularly as culture is oftentimes unrewarding (24).

This case report illustrates that, although abdominal pregnancies in rabbits may be associated with artificial insemination procedures in some farmed rabbits (12), they may also occur in wild $O$. cuniculus. This report also suggests that an abdominal pregnancy should be considered as a differential diagnosis in the investigation of a palpable abdominal mass or masses in O. cuniculus. Larger scale studies examining 
ectopic pregnancy in rabbits may reveal further insights into the pathogenesis of this condition.

\section{DATA AVAILABILITY}

All datasets analyzed for this study are included in the manuscript.

\section{ETHICS STATEMENT}

The animal study was reviewed and approved by University of Cambridge Department of Veterinary Medicine Ethics and Welfare Committee.

\section{AUTHOR CONTRIBUTIONS}

$\mathrm{KH}$ performed the gross and histological post mortem examination, conceived the study, collected and collated the data, and wrote the manuscript.

\section{REFERENCES}

1. Corpa JM. Ectopic pregnancy in animals and humans. Reproduction. (2006) 131:631-40. doi: 10.1530/rep.1.00606

2. Gauvin C, Amberger M, Louie K, Argeros O. Previously asymptomatic ruptured tubal ectopic pregnancy at over 10weeks' gestation: Two case reports. Case Rep Womens Health. (2019) 21:e00089. doi: 10.1016/j.crwh.2018.e00089

3. Stremick JK, Couperus K, Ashworth SW. Ruptured tubal ectopic pregnancy at fifteen weeks gestational age. Clin Pract Cases Emerg Med. (2019) 3:62-4. doi: $10.5811 /$ cpcem.2019.1.40860

4. Ge L, Sun W, Wang L, Cheng L, Geng C, Song Q, et al. Ultrasound classification and clinical analysis of ovarian pregnancy: a study of 12 cases. $J$ Gynecol Obstet Hum Reprod. (2019). doi: 10.1016/j.jogoh.2019.04.003. [Epub ahead of print].

5. Mouhajer M, Obed S, Okpala AM. Cervical ectopic pregnancy in resource deprived areas: a rare and difficult diagnosis. Ghana Med J. (2017) 51:94-7. doi: 10.4314/gmj.v51i2.8

6. Hong CC, Armstrong ML. Ectopic pregnancy in 2 guinea-pigs. Lab Anim. (1978) 12:243-4. doi: 10.1258/002367778781088567

7. Bland-Sutton J. Abdominal pregnancy in women, cats, dogs, and rabbits. Lancet. (1904) 164:1625-7.

8. Tena-Betancourt E, Tena-Betancourt CA, Zuniga-Munoz AM, HernandezGodinez B, Ibanez-Contreras A, Graullera-Rivera V. Multiple extrauterine pregnancy with early and near full-term mummified fetuses in a New Zealand white rabbit (Oryctolagus cuniculus). J Am Assoc Lab Anim Sci. (2014) 53:204-7.

9. Arvidsson A. Extra-uterine pregnancy in a rabbit. Vet Rec. (1998) 142:176.

10. Beddow BA. Ectopic pregnancy in a rabbit. Vet Rec. (1999) 144:624.

11. Turner PV, Brash ML, Smith DA. Rabbits. In: Turner PV, Brash ML, Smith DA, editors. Pathology of Small Mammal Pets. Hoboken, NJ: John Wiley \& Sons, Inc. (2018). p. 11.

12. Segura Gil P, Peris Palau B, Martinez Martinez J, Ortega Porcel J, Corpa Arenas JM. Abdominal pregnancies in farm rabbits. Theriogenology. (2004) 62:642-51. doi: 10.1016/j.theriogenology.2003.11.005

13. Marco-Jimenez F, Garcia-Dominguez X, Valdes-Hernandez J, Vicente JS. Extra-uterine (abdominal) full term foetus in a 15-day pregnant rabbit. BMC Vet Res. (2017) 13:307. doi: 10.1186/s12917-017-1229-7

14. Jacobson HA, Kibbe DP, Kirkpatrick RL. Ectopic fetuses in two cottontail rabbits. J Wildl Dis. (1975) 11:540-2.

15. Stott P, Wight N. Female reproductive tract abnormalities in European hares (Lepus europaeus) in Australia. J Wildl Dis. (2004) 40:696-703. doi: 10.7589/0090-3558-40.4.696

\section{FUNDING}

Publication costs were supported by Girton College, University of Cambridge. The funder had no role in study design, data collection and analysis, decision to publish, or preparation of the manuscript.

\section{ACKNOWLEDGMENTS}

The author thanks the pest controller who donates wild rabbit cadavers to the University of Cambridge for teaching and research purposes. The author gratefully acknowledges the technical expertise of Mrs. Debbie Sabin in the preparation of tissue sections. The rabbit described here was part of a post mortem study of the pathology of wild rabbits, and the author thanks the Ethics and Welfare Committee of the Department of Veterinary Medicine, University of Cambridge for their review of the study plan (reference: CR240).

16. Van der Merwe M, Racey PA. Abdominal foetus in the hare (Lepus timidus scoticus). J Zool. (1981) 197:309-11.

17. Holdt Somer SJ. Lithopedion: an unexpected finding. Am J Obstet Gynecol. (2016) 215:524 e521-2. doi: 10.1016/j.ajog.2016.06.056

18. Hughes K, Watson CJ. Sinus-like dilatations of the mammary milk ducts, Ki67 expression, and CD3-positive $\mathrm{T}$ lymphocyte infiltration, in the mammary gland of wild European rabbits during pregnancy and lactation. J Anat. (2018) 233:266-73. doi: 10.1111/joa.12824

19. Hughes K, Borman AM. Adiaspiromycosis in a wild European rabbit, and a review of the literature. J Vet Diagn Invest. (2018) 30:614-8. doi: $10.1177 / 1040638718772631$

20. Shin HY, Hennighausen L, Yoo KH. STAT5-driven enhancers tightly control temporal expression of mammary-specific genes. J Mammary Gland Biol Neoplasia. (2019) 24:61-71. doi: 10.1007/s10911-0189418-y

21. Weekers F, Van Herck E, Coopmans W, Michalaki M, Bowers CY, Veldhuis JD, et al. A novel in vivo rabbit model of hypercatabolic critical illness reveals a biphasic neuroendocrine stress response. Endocrinology. (2002) 143:764-74. doi: 10.1210/endo.143. 3.8664

22. Tao S, Dahl GE, Laporta J, Bernard JK, Orellana Rivas RM, Marins TN. Effects of heat stress during late gestation on the dam and its calf. J Anim Sci. (2019) 96:351. doi: 10.1093/jas/skz061

23. Simpson VR, Davison NJ, Dagleish MP. Causes of mortality and lesions observed post mortem in European moles (Talpa europaea) in Cornwall, South-west England. J Comp Pathol. (2019) 167:18-25. doi: 10.1016/j.jcpa.2018.11.006

24. Borman AM, Simpson VR, Palmer MD, Linton CJ, Johnson EM. Adiaspiromycosis due to Emmonsia crescens is widespread in native British mammals. Mycopathologia. (2009) 168:153-63. doi: $10.1007 /$ s11046-009-9216-6

Conflict of Interest Statement: The author declares that the research was conducted in the absence of any commercial or financial relationships that could be construed as a potential conflict of interest.

Copyright (C) 2019 Hughes. This is an open-access article distributed under the terms of the Creative Commons Attribution License (CC BY). The use, distribution or reproduction in other forums is permitted, provided the original author $(s)$ and the copyright owner(s) are credited and that the original publication in this journal is cited, in accordance with accepted academic practice. No use, distribution or reproduction is permitted which does not comply with these terms. 